\title{
PERFIL DE PORTADORES DE DIABETES MELLITUS TRATADOS NA ATENÇÃO BÁSICA DE ARAÇATUBA - SP: A IMPORTÂNCIA DA EDUCAÇÃO CRÍTICO-SOCIAL
}

\author{
Gean Domingos da Silva Souza \\ Débora Rossanese da Silva ${ }^{2}$ \\ Mariana Ferreira Sousa ${ }^{3}$ \\ Elton Carlos de Almeida ${ }^{4}$ \\ Sonia Maria Villela Bueno 5
}

SOUZA, G. D. da S.; SILVA, D. R. da; SOUZA, M. F.; ALMEIDA, E. C. de; BUENO, S. M. V. Perfil de portadores de Diabetes Mellitus tratados na atenção básica de Araçatuba - SP: a importância da educação crítico-social. Arq. Cienc. Saúde UNIPAR, Umuarama,v.18, n. 2, p, 101-105, maio/ago. 2014.

RESUMO: Investigar o perfil de pacientes diabéticos, em um grupo de pacientes atendidos na unidade de atenção básica de um município do interior paulista. Trata-se de uma pesquisa não experimental, de natureza quantitativa de caráter exploratório, descritivo, envolvendo 69 participantes de um universo de 670 cadastrados no programa HIPERDIA. Os dados foram analisados pela estatística descritiva. O estudo identificou que $64 \%$ pertenciam ao sexo feminino e $36 \%$ ao sexo masculino e que há uma predominância considerável prevalência do DM tipo 2 (88,4\%). A maioria dos pacientes investigados é portador de DM tipo 2 e grande parte desses sujeitos relatam saber o que é e como tratar a doença. Mas, carecem de conscientização sobre a doença, demandando efetiva educação para a melhoria da qualidade de sua vida. PALAVRAS-CHAVE: Diabetes mellitus; Enfermagem; Atenção primária à saúde.

\section{PROFILE OF DIABETES MELLITUS PATIENTS TREATED IN PRIMARY CARE IN ARAÇATUBA - SP: THE IMPORTANCE OF CRITICAL-SOCIAL EDUCATION}

\begin{abstract}
The aim of this paper is to investigate the profile of DM in a group of patients seen in a primary care unit in a city in the countryside of the state of São Paulo. This is a non-experimental, quantitative, exploratory and descriptive study, involving 69 participants from a universe of 670 patients enrolled in the HIPERDIA program. Data were statistically analyzed and evaluated using descriptive statistics. The study found that $64 \%$ of the patients were female and $36 \%$ male, as well as a predominance on the prevalence of type $2 \mathrm{DM}(88.4 \%)$. The majority of the patients investigated have type $2 \mathrm{DM}$ and most of these participants reported knowing what it is and how to treat the disease. However, they lack awareness of the disease, requiring effective education to improve their quality of life

KEYWORDS: Diabetes Mellitus; Nursing; Primary health care.
\end{abstract}

\section{Introdução}

O Diabetes Mellitus (DM) é uma doença que provoca grande impacto sobre a saúde populacional devido a sua alta prevalência, potencial gravidade e suas complicações. Classificada como uma doença metabólica, é caracterizada por hiperglicemia e associada a complicações, disfunções e insuficiência de vários órgãos, especialmente olhos, rins, nervos, cérebro, coração e vasos sanguíneos (SANTOS; SILVEIRA; CAFFARO, 2006). Resulta em uma afecção que pode provocar alterações da secreção ou ação da insulina nos tecidos corporais, envolvendo processos patológicos específicos como, por exemplo, a destruição das células beta do pâncreas (produtoras de insulina), resistência à ação deste hormônio ou distúrbios de sua secreção (BRASIL, 2006).

No DM tipo 1, ocorre destruição das células beta do pâncreas, usualmente por processo autoimune (tipo 1A) ou na forma idiopática (tipo 1B). De uma forma geral, a instalação do quadro de DM tipo 1 autoimune é relativamente, abrupta e muitas vezes o indivíduo pode identificar a data de início dos sintomas. DM tipo 2 é mais comum do que o tipo 1, perfazendo cerca de $90 \%$ dos casos de diabetes. Peculiarmente, no DM tipo 2, a incidência e a prevalência estão aumentando em proporções epidêmicas e atingindo a população na idade entre 30 a 69 anos (OMS, 2003).

De acordo com dados da rede de atenção básica no Brasil, é crescente a prevalência do DM e estima-se que, em 2025, haverá cerca de 11 milhões de pessoas com essa doença. Portanto, iniciativas para a promoção da saúde constituem uma das estratégias para sua prevenção e controle (BANDURA, 1997).

O controle glicêmico constitui um desafio, na medida em que depende da mudança de hábitos dos indivíduos portadores de DM. A troca de experiências entre os profissionais de saúde que lidam diretamente, com essa parcela da população e também entre indivíduos que convivem com DM, torna-se muito importante nas ações relacionadas às práticas cotidianas dos cuidados direcionados à doença (KNOWLER et al., 2002).

Os indivíduos com DM precisam estar conscientes dos sinais e sintomas e das alterações provocadas pela doença no organismo, além do conhecimento de seus fatores de risco, aprendendo sobre a doença, para serem capazes de tomar decisões diárias, de forma autônoma, para melhor evolução do tratamento e convívio com a doença. Assim, os profissionais de saúde que atuam no cuidado direto aos pacientes

OI: https://doi.org/10.25110/arqsaude.v18i2.2014.5174

${ }^{1}$ Enfermeiro. Doutorando do Departamento de Fisiologia da Faculdade de Medicina de Ribeirão Preto - Universidade de São Paulo. Email: geandomingos@ usp.br.

${ }^{2}$ Enfermeira pela Universidade Paulista. Email: debora-ros2011@hotmail.com

${ }^{3}$ Enfermeira pela Universidade Paulista. Email: marianaf.sousa@hotmail.com.

${ }^{4}$ Enfermeiro. Doutorando do Departamento de Enfermagem Psiquiátrica e Ciências Humanas da Escola de Enfermagem de Ribeirão Preto - Universidade de São Paulo. Email: ecarlos23@gmail.com.

${ }^{5}$ Educadora, Prof. ${ }^{\text {a Dr. }}{ }^{\text {, }}$ Associada III, Docente da EERP - Universidade de São Paulo. Email: smvbueno@eerp.usp.br. 
precisam ser capacitados para esse papel de educador, com conhecimento sólido da clínica, habilidades de comunicação, estratégias metodológicas e aplicabilidade de novas práticas. Para tanto, é preciso valorizar este processo estabelecendo vínculo de confiança com os pacientes (DM), possibilitando diálogo democrático, livre, aberto e franco com eles, favorecendo diversos esclarecimentos de dúvidas, neste sentido, para que conjuntamente, solucionem o problema em questão, visando sobremaneira, a otimização da vida (BUENO, 2009).

A adoção de medidas que incluem alteração dos hábitos cotidianos, dieta balanceada e prática de atividades físicas promovem um impacto muito positivo e essencialmente, significativo, para que o controle e o tratamento do DM sejam efetivos. Enfim, estudos demonstram cada vez mais, que o controle e a prevenção de complicações do diabetes são possíveis, por meio de programas educativos e efetivos (TORRES; HORTALE; SHALL, 2003; 2005)..$^{8-9}$

Baseados nas questões acima levantadas, o objetivo do presente estudo foi investigar o perfil, como também, conhecimento de portadores de DM, de um grupo de pacientes atendidos na atenção básica de um município do interior paulista.

\section{Material e Método}

Trata-se de uma pesquisa não experimental, de natureza quantitativa de caráter exploratório e descritivo, realizada em uma Unidade Básica de Saúde (UBS). Estudos não experimentais, servem para descrever, diferenciar ou examinar associações, ao contrário de procurar testar relação entre causa e efeito. Estudo exploratório descritivo, mostra o que existe de fato, determinam a frequência em que este fato ocorre e caracteriza a informação. A análise de dados do tipo quantitativo, é caracterizada por modos de inquirição sistemática preocupada com a compreensão dos seres humanos e da natureza de suas transações consigo mesmo e seus entornos (BREVIDEL, 2008).

A amostra por conveniência foi composta por 69 participantes de 670 pacientes portadores de DM cadastrados no programa HIPERDIA (Hipertensão e Diabetes) da UBS de um município do interior paulista onde foi realizado o estudo. A idade variou entre 24 a 80 anos. O período de coleta dos dados se fez entre janeiro a março do ano de 2012.

Os critérios para inclusão dos sujeitos da pesquisa foram: ser diabético, usuário e fazer acompanhamento regular, com assiduidade no programa HIPERDIA da UBS, ter maioridade e consentir participar do estudo.

A pesquisa teve início, somente, após aprovação pelo Comitê de Ética em Pesquisa da Universidade Paulista - UNIP (parecer 75156/2011) e autorização da Secretaria Municipal de Saúde do município onde foi realizada pesquisa seguindo todos os requisitos e preceitos éticos recomendados, conforme preconiza a Resolução 466/2012. A participação dos sujeitos se deu mediante a assinatura do Termo de consentimento livre esclarecido (TCLE) em duas vias, em que a primeira via ficou com os pesquisadores e a segunda, foi disponibilizada para os sujeitos participantes do estudo.

Os dados da pesquisa foram obtidos por meio da elaboração de um conjunto de questões, em forma de questionário, com respostas fechadas dicotômicas SIM ou não.
Dentre as principais questões levantadas no questionário, havia: Qual seu tipo de DM? Você sabe o que é DM? Há quanto tempo é portador? Sabe o que é Hiperglicemia e Hipoglicemia? Conhece as principais complicações do DM? Tem conhecimento de como tratar a doença?

Outras variáveis de interesse do estudo também foram abordadas como idade, sexo, período que desenvolveu a doença e suas complicações. A aplicação do questionário se deu concomitantemente, à entrega do TCLE, em horário matutino no qual os pacientes portadores de DM compareciam na UBS, para a realização do controle do Teste de Glicemia Capilar e respondiam após o teste. As entrevistas tiveram duração de 15 minutos. A aplicação dos questionários se dava uma vez por semana e teve uma duração de dois meses. Foram prestados esclarecimentos sobre os objetivos da pesquisa onde o anonimato das respostas e a participação facultativa, foram-lhes garantidos.

Após a aplicação dos questionários, os dados foram analisados e avaliados estatisticamente, expondo-os pelo percentual do que fora coletado efetuadas com o auxílio do software SPSS versão 13.0.

\section{Resultados}

Dos 670 portadores de DM cadastrados na UBS, participaram deste estudo, apenas 69 portadores, ou seja, $10 \%$ dessa população. A amostra identificou que $64 \%(n=44)$ pertenciam ao sexo feminino e $36 \%(n=25)$ do sexo masculino (Figura 1).

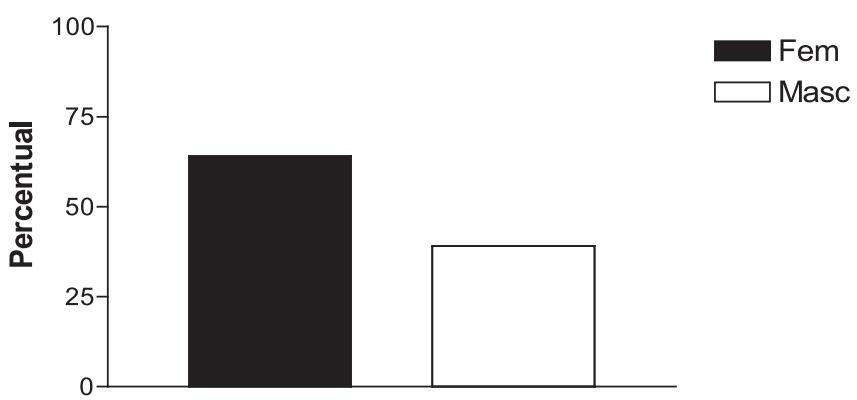

Figura 1: Distribuição percentual dos portadores de DM segundo gênero, Araçatuba/SP 2012.

Podemos observar também, que há uma predominância considerável em relação à prevalência de $88,4 \%$ $(n=61)$ portadores de DM tipo 2, e enquanto que $11,6 \%(n=8)$ são portadores de DM tipo 1 (Figura 2).

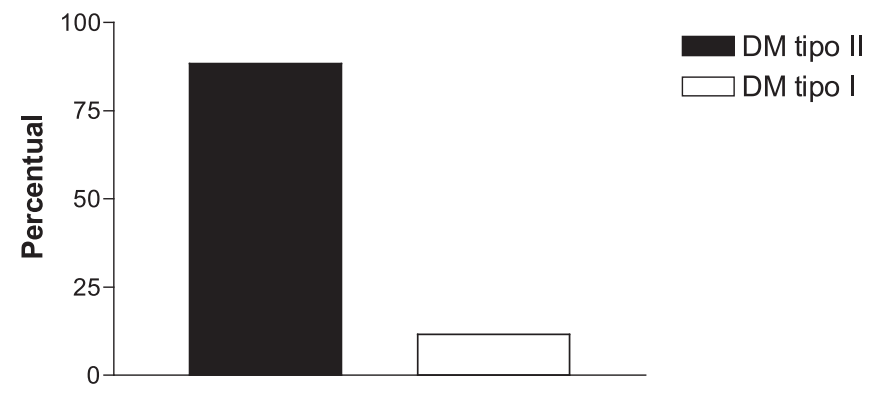

Figura 2: Distribuição percentual dos portadores pesquisados segundo Tipo de DM, Araçatuba/SP 2012.

Conforme a Tabela 1, a seguir, mostra as respostas de forma quantitativa, sobre o conhecimento dos principais 
eventos ocorridos nos portadores estudados, verifica-se $64 \%$ $(n=44)$ das mulheres e $36 \%(n=25)$ dos homens, conhecem a doença e sabem o que significa ser portador de DM. Com relação ao conhecimento do que é hipoglicemia, (ambos os sexos) apresentaram um percentual de 78,2\% $(n=54)$ de desconhecimento, o que também ocorreu nas respostas com re- lação à hiperglicemia 75,3\% $(n=52)$.

Em relação às complicações, entre o sexo masculino registrou-se o maior conhecimento $64 \%(n=16)$, já entre o sexo feminino $65,9 \%$ (29) responderam desconhecer as complicações da DM. Em relação ao tratamento da doença ambos os sexos sabem como trata-la 75,3\% (52).

Tabela 1: Conhecimento dos participantes sobre os principais eventos ocorridos, segundo gênero, Araçatuba/SP, 2012.

\begin{tabular}{lcccccccc}
\hline Conhecimento & \multicolumn{3}{c}{ Masculino } & \multicolumn{5}{c}{ Feminino } \\
\hline & Sim & $\%$ & Não & $\%$ & Sim & $\%$ & Não & $\%$ \\
\hline O que é DM & 18 & 72 & 7 & 28 & 25 & 57 & 19 & 43 \\
Hipoglicemia & 9 & 36 & 16 & 64 & 6 & 14 & 38 & 86 \\
Hiperglicemia & 10 & 40 & 15 & 60 & 7 & 16 & 37 & 84 \\
Conhece que pode resultar em complicações & 16 & 64 & 9 & 36 & 25 & 34 & 29 & 66 \\
Como tratar a doença & 19 & 76 & 6 & 24 & 33 & 75 & 11 & 25 \\
\hline
\end{tabular}

A Tabela 2 a seguir, mostra as respostas dos sujeitos no que que se refere ao tempo de conhecimento do diagnóstico da doença, levando-se a depreender que o período de 02 a 05 anos, para ambos os sexos, foi o que apresentou um maior percentual $70,1 \%(n=24)$, ao informarem sobre o tempo que tinham de conhecimento sobre a sua doença.

Tabela 2: Tempo de conhecimento do diagnóstico de DM dos participantes, Araçatuba/SP, 2012.

\begin{tabular}{lcccc}
\hline & \multicolumn{2}{c}{ Masculino } & \multicolumn{2}{c}{ Feminino } \\
\cline { 2 - 5 } Tempo do diagnóstico & $\mathbf{N}$ & $\mathbf{\%}$ & $\mathbf{N}$ & $\mathbf{\%}$ \\
\hline Até um ano & 2 & 8 & 12 & 27,3 \\
02 a 05 anos & 9 & 36 & 15 & 34,1 \\
06 a 10 anos & 5 & 20 & 8 & 18,2 \\
> 10 anos & 9 & 36 & 9 & 20,4 \\
Total & 25 & 100 & 44 & 100 \\
\hline
\end{tabular}

\section{Discussão}

Atualmente, devido às alterações do perfil epidemiológico da população, tornam-se relevantes as mudanças impactantes sobre a assistência em saúde da população, para alcançar os objetivos de atenção desejadas, fazendo-se necessário, priorizar algumas ações relacionadas com a promoção da saúde e prevenção de danos decorrentes das doenças, principalmente, as que são evitáveis.

As intervenções educativas são essenciais nesse contexto de atendimento, com vistas à inclusão das pessoas no cuidado da própria saúde, e para que tal objetivo seja alcançado, há urgência do entendimento de como a população percebe-se doente e lida com suas questões de saúde/doença (MENDES, 2002; THORNE, 2008; SILVA et al., 2009; FREITAS, ARCIA, 2012).

Em relação ao gênero (Tabela 1) observamos que há maior prevalência de DM entre as mulheres (64\%) quando comparado aos homens (36\%). De acordo com um estudo transversal realizado com microdados da Pesquisa Nacional por Amostra de Domicílios (PNAD) no período 1998-2008, o coeficiente de prevalência de diabetes elevou-se significativamente a partir da faixa etária de 40 a 49 anos, sendo maior ainda, na faixa etária de 70 a 79 anos, para ambos os sexos.
No presente estudo, mostra-se maior prevalência entre mulheres, corroborando com trabalhos atuais da literatura. No entanto, alguns estudos atuais, já observam um aumento da prevalência entre os homens, notando-se que essa elevação de 2,6\%, em 1998, para 3,8\%, em 2008, confirma tal prevalência (HARRIS, 1998). Enquanto as condições crônicas prevalecem, as ações de saúde são essencialmente curativas, voltadas para o atendimento de agravos agudos. Porém, uma visão assistencial preventiva focada na promoção da saúde deve ser cada vez mais priorizada, nos tempos atuais, alicerçada em uma educação conscientizadora fundamentada na liberdade e na transformação do cidadão e da sua própria realidade, de forma crítico-reflexiva (BUENO, 2009).

A crescente redução da atividade física, agitação do mundo globalizado e capitalizado entre outros fatores favorecidos pela vida pós-moderna e na busca incessante pelo desenvolvimento no mundo contemporâneo, vem contribuindo severamente para o surgimento de diversas doenças crônicas. Algumas evidências sugerem que o sedentarismo é um fator de risco, seriamente, contribuinte para DM (SANTOS; SILVEIRA; CAFFARO, 2006).

Em relação aos tipos de DM encontradas em nossos estudos, constatou-se um maior número de casos de DM tipo $2(88,4 \%)$. Dados semelhantes foram encontrados em outro estudo (ORTIZ; ZANETTI, 2001). O crescente aumento na taxa de sobrepeso e obesidade torna-se relevante para o aumento da prevalência do DM tipo 2. Resultados de diversos inquéritos populacionais mostram que a incidência desse tipo 2, aumenta com a média do peso da população (ORTIZ; ZANETTI, 2001). A literatura descreve que o diagnóstico de DM tipo 2 está se manifestando mais precocemente, e vêm sendo observado até mesmo na adolescência (HARRIS, 1998).

Os aumentos no índice da prevalência de DM, têm sido associados às rápidas transformações demográficas e socioeconômicas observadas entre vários países em desenvolvimento. A grande variação da prevalência do diabetes em diferentes nações tem sido atribuída a uma combinação de diferenças genéticas e fatores ambientais como dieta, obesidade, sedentarismo e desenvolvimento intrauterino (POPKIN et al., 1995; CAVALI-SFORZA et al., 1996; ZIMMET; CARTY; COURTEN, 1997). A inatividade física quanto à dieta inadequada na etiologia da obesidade possui uma relação direta e positiva, com o aumento da incidência do 
$\mathrm{DM}$, independente de fatores genéticos ou hereditariedade e do índice de massa corpórea (IMC) (MANSON et al., 1991; PRENTICE; JEBB, 1995; ZIMMET; CARTY; COURTEN, 1997).

Quando questionados acerca do conhecimento sobre os principais eventos ocorridos (Tabela 1) foi constatado que os portadores de DM, de ambos os sexos, não têm conhecimento sobre o que é hipoglicemia e hiperglicemia. No entanto, o conhecimento dos sintomas, torna-se muito importante, pois os indivíduos com DM mal controlados, são mais suscetíveis às infecções, devido à gravidade do déficit de insulina e as alterações observadas no sistema imune desses indivíduos (SANTOS; SILVEIRA; CAFFARO, 2006).

A maneira como se dá a convivência e o enfrentamento dos portadores de DM no cotidiano, bem como os fatores relacionados ao tratamento, seja ele medicamentoso ou mudanças nos hábitos alimentares, nota-se que as principais diferenças entre um bom convívio com a doença, são observados quando os portadores conhecem melhor a doença, visto que assim ocorre a desmistificação acerca do DM, bem como as estratégias para lidar com a doença, auxílio no contexto familiar e social (POPKIN et al., 1995).

Nota-se então, que todas essas variáveis, influenciam diretamente, na adesão ao tratamento de DM e constituem elementos relevantes que devem ser considerados no planejamento de ações e intervenções mais eficazes (OLIVEIRA et al., 2011).

Assim, observamos que a maioria dos pacientes (34\%) obtiveram o diagnóstico entre 02 a 05 anos. Analisados os dados relacionados à idade média em que o portador de DM descobriu a doença, constatou-se aqui, que o conhecimento sobre o diagnóstico permanece entre 60 a 69 anos de idade, representando $68,1 \%(n=47$, dados não mostrados em tabela). Esta faixa etária é dominante para ambos os sexos. Pesquisa semelhante que estudou o perfil dos portadores de DM, também observou resultado semelhante (GUIMARÃES; TAKAYANAGUI, 2002).

\section{Conclusão}

Os dados encontrados nesta investigação, concluem que a maioria dos pacientes investigados são portadores de DM, tipo 2. Constatou-se também, que a maioria dos sujeitos relatou saber o que é DM e de como tratar a doença (ambos os sexos).

Os achados merecem ser melhor analisados pelos profissionais de saúde, dentro de uma abordagem mais multiprofissional possível, visando à implementação de programas educativos na área de atuação da atenção básica, visando portanto, a auxiliar na promoção da saúde e na prevenção das complicações e impacto provocado pelas doenças crônico-degenerativas na saúde da população e no serviço de atenção à saúde, em particular o DM, por meio da utilização das mais diferentes abordagens.

Estudos sobre os conhecimentos dos portadores de DM são muito importantes, visto que as intervenções para o controle dessa doença na população adulta sejam cada vez mais efetivas, contribuindo pois, para os programas de saúde vigentes na atenção básica, voltados para uma educação mais aberta e conscientizadora, que permeia a participação ativa do cliente e nas decisões que venha culminar com a melhoria da qualidade de vida de si e de toda a coletividade. Assim, depreendemos estudos que avaliam o perfil dos pacientes diabéticos podem auxiliar na prevenção de complicações provocadas pelo DM, aumentando então, o nível de qualidade de vida tanto física quanto mental.

\section{Referências}

BANDURA, A. Self-effi cacy: toward a unifying theory of behavioral change. Psychol Rev. v. 84, n. 2, p.191-215, 1977.

BRASIL. Ministério da Saúde. Secretaria de Atenção à Saúde. Departamento Atenção Básica. Diabetes Mellitus. Cadernos de Atenção Básica - nº 16 Série A. Normas e Manuais Técnicos. Brasília - DF; 2006.

BREVIDEL, M. M. Guia Prático para Docentes e Alunos da Área da Saúde. 2. ed. São Paulo: Itária, 2008.

BUENO, S. M. V. Tratado de educação preventiva em sexualidade, DST-aids, drogas e violência nas escolas. 1. ed. Ribeirão Preto: Editora FIERP, 2009.

CAVALLI-SFORZA, L. T. et al. Nutritional aspects of changes in disease patterns in the Western Pacific Region. Bulletin of the World Health Organization. v. 74, p. $307-$ 318, 1996.

FREITAS, L. R. S.; ARCIA, L. P. Evolution of prevalence of diabetes and associated hypertension in Brazil: analysis of National Household Sample Survey, 1998, 2003 and 2008. Epidemiol Serv Saúde. v. 21, n. 1, p. 07-19, 2012.

GUIMARÃES, F. P. M.; TAKAYANAGUI, A. M. M. Orientações recebidas do serviço de saúde por pacientes para o tratamento do portador de diabetes mellitus tipo 2 . Rev Nutr, v.15, v. 1, p. 37-44, 2002.

HARRIS, M. I. Diabetes in America: Epidemiology and scope of the problem. Diabetes Care. v. 21, n. 3, p. 11-4, 1998.

KNOWLER, W. C. et al. Diabetes Prevention Program Research Group. Reduction in the incidence of type 2 diabetes with lifestyle intervention or metformin. N Engl J Med. v. 346, n. 6, p. 393-403, 2002.

MANSON, J. E. et al. Physical activity and incidence of noninsulin-dependent diabetes mellitus in women. Lancet, v. 338, p. 774-8, 1991.

MENDES, E. V. A atenção primária à saúde no SUS. Fortaleza: Escola de Saúde Pública do Ceará, 2002.

OLIVEIRA, N. F. et al. Diabetes Mellitus: desafios relacionados ao autocuidado abordados em Grupo de Apoio Psicológico. Rev bras Enferm, v. 64, n. 2, p. 301-7, 2011.

OMS. Organização Mundial da Saúde. Cuidados inovadores para condições crônicas: componentes estruturais de ação: 
relatório mundial. Brasília: Organização Mundial da

Saúde; 2003.

ORTIZ, M. C. A.; ZANETTI, M. L. Levantamento dos fatores de risco para diabetes mellitus tipo $2 \mathrm{em}$ uma instituição de ensino superior. Rev Latino Am Enferm. v. 9, n. 3, p. 58-63, 2001.

POPKIN, B. M. et al. Review of dietary and environmental correlates of obesity with emphasis on developing countries. Obesity Research, v. 3, n. 2, p. 145-53, 1995.

PRENTICE, A. M.; JEBB, S. A. Obesity in Britain: Gluttony or sloth? BMJ, v. 311, p. 437-439, 1995.

SANTOS, V. P.; SILVEIRA, D. R.; CAFFARO, R. A. Fatores de risco para amputações maiores primárias em pacientes diabéticos. J Méd. v. 124, n. 2, p. 66-70, 2006.

SILVA, K. L. et al. Educação em Enfermagem e os desafios para a promoção de saúde. Rev Bras Enferm. v. 62, n. 1, p. 86-91, 2009.

THORNE, S. Chronic disease management: what is the concept? Can J Nurs Res. v. 40, n. 3, p. 7-14, 2008.

TORRES, H. C.; HORTALE, V. A.; SHALL, V. T. A experiência de jogos em grupos operativos na educação em saúde para diabéticos. Cad Saúde Pública. v. 19, n. 4 p. 1039-47, 2003

TORRES, H. C.; HORTALE, V. A.; SHALL, V. T. Validação dos questionários de conhecimento (DKN-A) e atitude (ATT-19) de Diabetes Mellitus. Rev Saúde Pública. v. 39 , n. 6 , p. $906-11,2005$.

ZIMMET, P. Z.; CARTY, D. J.; COURTEN, M. P. The global epidemiology of non-insulin-dependent diabetes mellitus and the metabolic syndrome. Journal of Diabetes and Its Complications. v. 11, n. 2, p. 60-8, 1997. 